\title{
Photometry/Spectrum Analysis, Visible Light
}

National Cancer Institute

\section{Source}

National Cancer Institute. Photometry/Spectrum Analysis, Visible Light. NCI Thesaurus.

Code C18938.

Analysis of the absorption or transmittance of light in the visible region of the electromagnetic spectrum by a specific compound. By itself, this technique does not lead to the identification of a substance, but analysis of the spectrum (absorption versus wavelength) can help identify the presence of specific chromophores in the molecule. When the identity of the compound is known, determination of the absorbance at a specific wavelength yields quantitative information about the material analyzed. 